\title{
The prevalence of vascular injury utilising the lateral parapatellar approach for malignant distal femoral tumour resections: a case series
}

\author{
${ }^{1}$ Department of Orthopaedics, University of Pretoria, Pretoria, South Africa \\ ${ }^{2}$ Department of Orthopaedics, 1 Military Hospital, University of Pretoria, Pretoria, South Africa \\ ${ }^{3}$ Department of Orthopaedics, Kalafong Hospital, University of Pretoria, Pretoria, South Africa \\ ${ }^{4}$ Clinical Psychologist, Pretoria East Hospital, Pretoria, South Africa \\ *Corresponding author: nvanderwatt@gmail.com
}

Nicolaas P van der Watt, ${ }^{1 *}$ (D) Odette Koch, ${ }^{2}$ Theo LB le Roux, ${ }^{2}$ Jannes G Meijer, ${ }^{3}$ Heather McLoughlin ${ }^{4}$

Citation: Van der Watt NP, Koch O, Le Roux TLB, Meijer JG, McLoughlin $\mathrm{H}$. The prevalence of vascular injury utilising the lateral parapatellar approach for malignant distal femoral tumour resections: a case series. SA Orthop J 2021;20(3):147-150. http://dx.doi.org/10.17159/23098309/2021/v20n3a2

Editor: Dr Thomas Hilton, University of Cape Town, Cape Town, South Africa

Received: June 2020

Accepted: December 2020

Published: August 2021

Copyright: (c) 2021 Van der

Watt NP. This is an open-access article distributed under the terms of the Creative Commons Attribution Licence, which permits unrestricted use, distribution and reproduction in any medium, provided the original author and source are credited.

Funding: No funding was received for this study.

Conflict of interest: The authors declare they have no conflicts of interest that are directly or indirectly related to the research.

\begin{abstract}
Background

Distal femoral tumour resections are mostly performed through a medial or anteromedial approach. The lateral parapatellar approach is an alternative method. This case series assessed vascular complications during the resection of malignant distal femoral tumours via the lateral parapatellar approach.
\end{abstract}

\section{Methods}

A retrospective case series at a private practice in Pretoria was performed. All patients who underwent malignant distal femoral tumour resections through a lateral parapatellar approach between 2001 and 2019 were included in the study. All cases were performed by a single surgeon. An analysis of the patients' files was performed, to determine if there were any intraoperative or immediately postoperative vascular complications.

\section{Results}

Thirty-six patients were identified who underwent resection of their malignant distal femoral tumours via the lateral parapatellar approach. Osteosarcoma was the most prevalent bone tumour $(81 \%)$. All resection margins were clear on histology reports. The vascular complication rate was $3 \%(95 \% \mathrm{Cl} 0-8 \%)$. Twelve patients demised over the 18 -year period $(33 \%)$.

\section{Conclusion}

The findings suggest that a low risk of vascular complications can be expected when resecting malignant distal femoral tumours through a lateral parapatellar approach. This rate of vascular injury is comparable to other studies that also performed distal femoral tumour resections through other approaches.

Level of evidence: Level 4

Keywords: malignant distal femoral tumours, lateral parapatellar approach, vascular complications, tumour resection, endoprosthesis

\section{Introduction}

Malignant bone tumours of the distal femur often abut and at times even encase surrounding neurovascular structures. Due to the hypervascularity associated with these malignant bone tumours, vascular complications of dissection may include vessel laceration, venous or arterial intimal damage, arterial thrombosis with resultant limb ischaemia or venous thrombosis with possible thromboembolic events. ${ }^{1}$ Surgery of malignant bone tumours has developed significantly over time but requires a high level of skill. It is a great responsibility for the tumour surgeon to provide a functional solution to the patient in the presence of such a devastating diagnosis. Amputation of a limb would have been accepted as an appropriate outcome in the past; however, the aim is now to salvage the limb and ultimately improve the patient's functionality, satisfaction and quality of life. ${ }^{2}$ Myers et al. have shown that limb salvage is more cost-effective when compared to amputation in the long run. ${ }^{3}$

Different surgical approaches to access the distal femur have been described. The most often used surgical approach and gold standard of accessing the distal femur for tumour resection is the anteromedial approach. This approach exposes the anterior aspect of the femur and gives access to the popliteal fossa. It identifies the neurovascular bundle in Hunter's canal and allows it to be mobilised and protected throughout the procedure. With this approach, additional soft tissue cover is seldom needed during distal femur resections; soft tissue cover is, however, frequently 
indicated during proximal tibia resections. ${ }^{4}$ The anteromedial approach is advised for experienced surgeons in the field of tumour and sepsis surgery.

The lateral approach to the femur is the most used approach relating to benign bone lesions in the distal femur. It is considered to be surgically less demanding as there is no need for neurovascular dissection. The option of proximally extending the incision is readily available and holds benefits for future surgery. ${ }^{4}$

In the series described in this article, the lateral parapatellar approach was routinely used for the biopsy and resection of distal femoral tumours. There is theoretically an increased risk for vascular injury with these procedures, due to the proximity of the vascular structures and due to the neo-vascularisation associated with malignancy in bone. There is limited data in the literature regarding the incidence of vascular injuries when the lateral parapatellar approach to the distal femur for tumour resections is used..$^{5-7}$ The available literature mostly reviews patient outcomes and endoprosthetic survival. The rarity of these procedures is confirmed by the long-term follow-up and extended time frames in which results were recorded. $2,3,8,9$

This study aimed to determine the prevalence of intraoperative and immediate postoperative vascular complications when resecting distal femoral tumours through a lateral parapatellar approach. It is based on the third author's experience (TLBR) with 36 consecutive patients who had distal femoral tumour resections through a lateral parapatellar approach.

\section{Materials and methods}

A retrospective case series at a private orthopaedic practice in Pretoria was conducted. Ethical approval was obtained prior to the

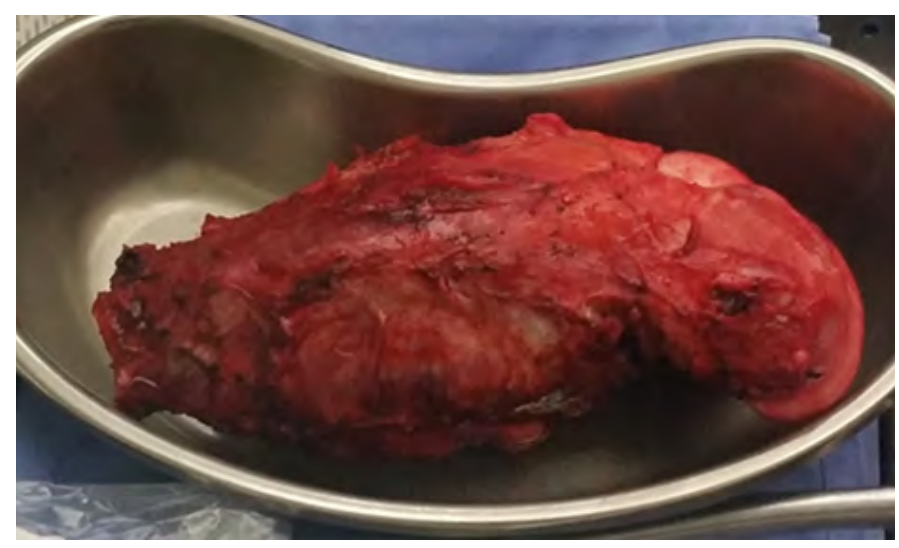

Figure 1. Illustration showing an osteosarcoma of the distal femur in a 13-year-old male, after a distal femoral tumour resection via a longitudinal lateral parapatellar approach

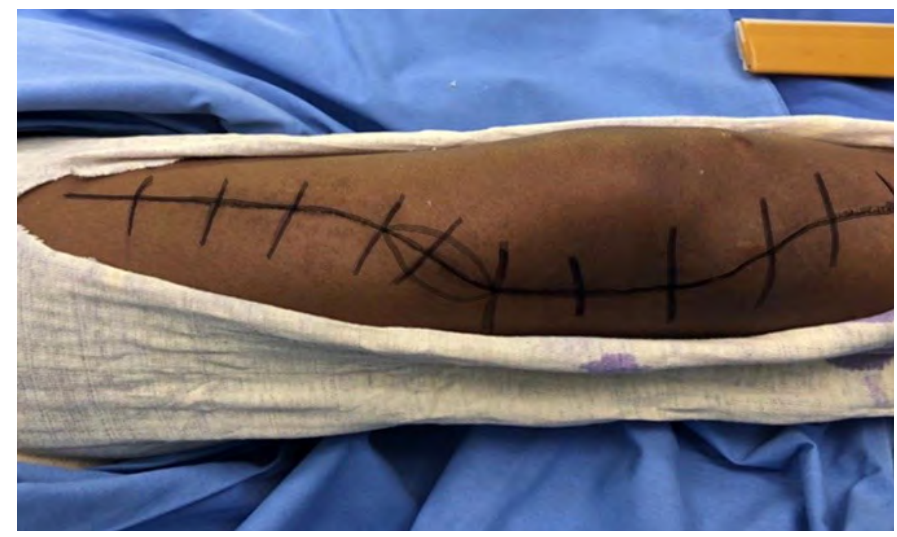

Figure 2. Illustration showing the markings of the longitudinal lateral parapatellar approach, that can be extended as far proximally as the anterior superior iliac spine (note: ellipse excision of biopsy site) commencement of data collection from patient records. All cases performed between January 2001 and July 2019 were scrutinised. Patients included in the study were those that had a malignant primary or metastatic distal femoral tumour lesion that was resected (Figure 1), and a prosthesis inserted through the lateral parapatellar approach (Figure 2). An experienced tumour surgeon performed all cases. Complete records of cases were mandatory with a minimum follow-up of six months. Patients were excluded from the study if a distal femoral tumour resection was done via any approach other than the lateral parapatellar approach and if the extent of the tumour resulted in a non-salvageable resection. Benign distal femoral tumours were excluded from the study. All tumour resections in this series were performed via a longitudinal lateral parapatellar approach to the femur. All resected tumours were sent for histological analysis. The patients were reviewed daily by the same surgeon until discharge from hospital. They were then followed-up at two weeks and six weeks postoperatively. Treatment in the oncology unit continued as per protocol.

\section{Surgical procedure}

The surgical procedure is routinely performed in the supine position. The foot is placed on a bolster, and the thigh is supported against a limb positioner. The patient's MRI is used to determine the level of the resection on the femur. The affected limb is draped with the Charnley double-drape technique and prepared from the iliac crest to the foot. No tourniquet is used, and preoperative antibiotics are administered. The surgical incision is marked, including an ellipse around the previous biopsy area on the lateral aspect of the knee (Figure 2). The knee is flexed, and this position allows for the soft tissue and vascular structures in the popliteal fossa to 'fall with gravity' away from the surgical area. A longitudinal incision is made in the midline from the tibial tubercle and extended as proximal as needed, utilising a lateral parapatellar approach and dislocating the patella medially. The femur is measured and marked at the level of the resection with a constant reference point on the tibia. This pre-resection measuring must be accurate as the aim is to restore the leg length when inserting the prosthesis. The incision can be extended proximally to the tip of the greater trochanter or the anterior superior iliac spine in cases where a

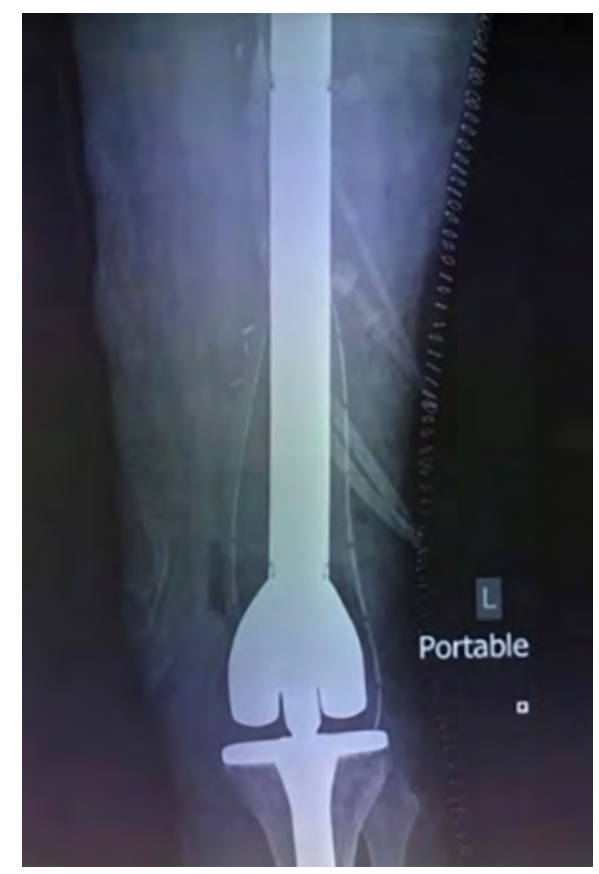

Figure 3. Illustration showing an anteroposterior radiograph of a total femur endoprosthesis performed utilising the lateral parapatellar approach 
total femur endoprosthesis is inserted (Figure 3). An elliptical resection of the lateral biopsy site is performed with extension to the underlying tumour, including a $2 \mathrm{~cm}$ margin in all planes. This crucial part of the procedure emphasises the importance of placing the biopsy tract accurately in order to prevent tumour spillage. The skin, subcutaneous tissue and fascia lata are incised in line with its fibres. The perforators are ligated and managed. The vastus lateralis can be transposed anteriorly over the femur after all perforators are tied off. A margin of muscle can be left on the tumour for histological purposes, and the entire length of the femur can be exposed. The cruciate ligaments, collateral ligaments and posterior capsule are cut and the specimen is dissected from distal to proximal. Particular attention is paid to the artery when the tumour is excised as it can be pulled towards the tumour. The vascular structures in Hunter's canal are not routinely identified on the medial aspect. The femur is resected at the predetermined level, and a sample of the proximal medullary canal is taken with clean instruments. A pathologist performs a frozen section on the specimen of the medullary canal to confirm tumour-free margins. The dorsalis pedis pulse is routinely checked at this stage of the surgery. After a glove change, the tibia is prepared using routine steps with clean instruments, while awaiting the pathology report. The procedure continues in a stepwise fashion after that. The wound is closed routinely in layers over a drain. All patients are observed in a high care facility postoperatively to optimise pain management and close monitoring of the limb. The same histopathologist who performed the frozen section is responsible for the formal pathology report of the excised tumour.

\section{Statistical analysis}

Continuous variables were described using mean and median with a range. Categorical variables were described using frequency and proportions. The rate of complications was expressed as a proportion of all cases with a $95 \%$ confidence interval $(\mathrm{Cl})$. All analyses were conducted in Excel 2013.

\section{Results}

Thirty-six patients over an 18-year period were included in the study. The study population consisted of 23 male and 13 female patients. The median age was 23.1 years (7-69 years) with interquartile range (IQR) of $14-38$ years, mean=27.2 years, $\mathrm{SD}=17.7$. The median time of follow-up was 19.4 months (6-94 months), IQR=10-41 months, mean=29.7 months, $S D=25.7$. Nine patients were still being actively followed up at the time of this report. Three patients had their follow-up elsewhere after they completed their six-months follow-up at the practice. Histologically, the majority of tumours were osteosarcoma (29/36; 81\%) (Table I). All resection margins on the final histological reports were clear. No patients required additional soft tissue cover. Over the 18 years, 12 patients died $(33 \%)$.

One vascular complication was recorded during the study period. An overall vascular complication rate of $3 \%(95 \% \mathrm{Cl} 0-8 \%)$ was thus reported. The vascular injury occurred in a 47-year-old male who presented with telangiectatic osteosarcoma. The vascular injury was recognised intraoperatively; the artery was injured on the medial border of the distal femur due to tumour displacement. An immediate arterial repair was performed by a general surgeon on call for the hospital. Limb perfusion was, however, inadequate as measured by Doppler flow studies. The patient was transferred to a specialist vascular surgeon, and a femoral-popliteal bypass procedure was performed within 12 hours of the injury. The limb was salvaged. The patient died six years later due to metastatic disease.

\section{Discussion}

Malignant primary bone tumours occur most commonly in the distal femoral area and in the proximal tibia. ${ }^{4}$ Bone sarcomas account for $0.2 \%$ of all malignancies with an adjusted incidence rate of 0.9 per 100000 per year for all bone and malignant joint tumours. ${ }^{10}$

Primary bone tumours are a scarce entity. Distal femoral tumours predominantly consist of osteosarcoma, chondrosarcoma and Ewing's sarcoma.11,12 Neo-adjuvant chemotherapy, surgical resection with a wide margin and adjuvant chemotherapy is considered to be the mainstay of treatment of osteosarcoma and Ewing's sarcoma. ${ }^{13}$ Reconstructive options have evolved significantly and differ for various age groups. These include osteoarticular allograft, allograft arthrodesis, prosthetic arthrodesis, rotationplasty and endoprosthetic replacement surgery. ${ }^{1}$ Each tumour resection is individualised with regard to its location, histological type and extension into the soft tissues. $3,4,9$

In this series, $81 \%$ of the tumours resected were osteosarcoma, of which conventional osteosarcoma was the most prevalent. A recent local paper also reported that osteosarcoma accounted for $72.6 \%$ of all primary malignant bone tumours, of which the distal femur was the most common site $(44.7 \%)$ with a slight male predominance. The average age in this study population was 27 years (range 7-69) and the mean age of diagnosis in the osteosarcoma group was 20.1 years (range 7-47). This is in keeping with findings in the literature, which shows a higher prevalence of osteosarcoma in the second decade of life. ${ }^{11}$ Results in this series did not reflect a bimodal distribution. Contrary to the literature, which shows a slight male predominance, this series had a slight female-to-male predominance ratio of 1.1:1.,111,14 Telangiectatic osteosarcoma represents $3-10 \%$ of all osteosarcoma. ${ }^{15}$ The age distribution for this subtype of osteosarcoma tends to be younger than conventional osteosarcoma. ${ }^{15,16}$ In this series, the patient who had the vascular injury had a telangiectatic osteosarcoma, which is rare at the age of 47 years. The series described in this article had a vascular complication rate of $3 \%(95 \% \mathrm{Cl} 0-8 \%)$. This complication rate is higher than the reported incidence rate found in a large study done by Natarajan et al. In their series of 246 patients, they had one vascular complication which led to an amputation. Their complication rate of $0.4 \%$ is lower than the current series and could be due to the large series they examined

Table I: Description of tumour analysis and vascular complications

\begin{tabular}{|c|c|c|c|c|c|}
\hline Tumours & Patients & Male/Female & Tumour margin & Vascular injury & Mean age (years) \\
\hline \multicolumn{6}{|l|}{ Osteosarcoma } \\
\hline Conventional & $26(72 \%)$ & $12 / 14$ & Complete & 0 & 17 \\
\hline Telangiectatic & $2(6 \%)$ & $2 / 0$ & Complete & 1 & 29 \\
\hline Parosteal & $1(3 \%)$ & $0 / 1$ & Complete & 0 & 16 \\
\hline Chondrosarcoma & $5(14 \%)$ & $0 / 5$ & Complete & 0 & 59 \\
\hline \multicolumn{6}{|l|}{ Metastatic disease } \\
\hline Renal clear cell ca & $1(3 \%)$ & $1 / 0$ & Complete & 0 & 48 \\
\hline B-cell lymphoma & $1(3 \%)$ & $1 / 0$ & Complete & 0 & 68 \\
\hline Total & 36 & & & 1 & \\
\hline
\end{tabular}


as well as the fact that they included benign and malignant bone tumours. ${ }^{8}$ A comparison of vascular complications pertaining to the lateral parapatellar approach could not be made due to the paucity in literature regarding this approach.

Accardo and colleagues investigated the outcomes of a quadriceps-sparing lateral approach to the distal femur for tumour resection and reported no vascular complications when the lateral approach was used. They stated that an added benefit to this approach was that the incision can be extended to the proximal femur to provide improved exposure if needed. ${ }^{17}$

All tumours were successfully resected with the approach used in this study and the complete resections were confirmed by the final histology report. There was no need for additional soft tissue cover or local flaps in this series. When the anteromedial approach was used, Bickels et al. reported the need for 25 gastrocnemius flaps in a series of 110 patients, and Capanna et al. reported the need for rotational or free flaps in three of their 14 patients utilising either an anteromedial or anterolateral approach. ${ }^{2,6}$

The main limitation of this study is its retrospective nature. All patients were operated by a single surgeon from a single institution and could therefore be subjected to bias. The small sample size of 36 patients being operated over an 18-year period is considered another limitation. There was no control group in this study. Other outcome measures such as wound complications, patient outcome and functionality scores were not assessed.

\section{Conclusion}

In this series, all distal femoral tumours were accessible and completely resected via the lateral parapatellar approach. The approach avoids dissection of the neurovascular bundle by staying lateral to the bundle, which reduces the risk of iatrogenic injury to vascular structures. This approach had a low vascular complication rate and proved to be safe and reliable. It should be in the orthopaedic surgeon's armamentarium when resecting malignant bone tumours of the distal femur.

\section{Ethics statement}

The authors declare that this submission is in accordance with the principles laid down by the Responsible Research Publication Position Statements as developed at the 2nd World Conference on Research Integrity in Singapore, 2010.

Ethical approval was obtained from the Research Ethics Committee of the University of Pretoria prior to the commencement of data collection (546/2019).

All procedures were in accordance with the ethical standards of the responsible committee on human experimentation (institutional and national) and with the Helsinki Declaration of 1975 , as revised in 2008.

\section{Declaration}

The authors declare authorship of this article and that they have followed sound scientific research practice. This research is original and does not transgress plagiarism policies.

\section{Author contributions}

NPW: Study conceptualisation, data capture, data analysis and interpretation, first draft preparation, manuscript preparation and revision

OK: Study conceptualisation, data analysis and interpretation, manuscript review

TLBR: Study conceptualisation, manuscript review

JGM: Data capture, draft preparation, manuscript preparation and revision

HM: Data capture, data analysis

\section{ORCID}

NP van der Watt (iD https://orcid.org/0000-0002-0706-2787

O Koch (iD https://orcid.org/0000-0003-1871-9569

TLB le Roux (D) https://orcid.org/0000-0003-1871-9569

JG Meijer (iD) https://orcid.org/0000-0001-7773-6081

H McLoughlin (iD https://orcid.org/0000-0001-8330-6563

\section{References}

1. Yang R-S. Endoprosthesis-related complications after limb-salvage operation of malignant bone tumors around the knee. Biomed Eng. 2004;16(03):133-42. https://doi.org/10.4015/S1016237204000189.

2. Bickels J, Wittig JC, Kollender Y, et al. Distal femur resection with endoprosthetic reconstruction: A long-term followup study. Clin Orthop Relat Res. 2002;(400):225-35.

3. Myers GJC, Abudu AT, Carter SR, Tillman RM, Grimer RJ. Endoprosthetic replacement of the distal femur for bone tumours. J Bone Joint Surg Br. 2007;89(4):521-26.

4. Malawer MM, Wittig JC, Bickels J, Wiesel SW. Operative techniques in orthopaedic surgical oncology. 2nd ed. Philadelphia: Wolters Kluwer; 2016.

5. Biau D, Faure F, Katsahian S, et al. Survival of total knee replacement with a megaprosthesis after bone tumor resection. J Bone Joint Surg Am. 2006;88(6):1285-93

6. Capanna R, Scoccianti G, Campanacci DA, et al. Surgical technique: Extraarticular knee resection with prosthesis-proximal tibia-extensor apparatus allograft for tumors invading the knee. Clin Orthop Relat Res. 2011;469(10):2905-14. https://doi.org/10.1007/s11999-011-1882-2.

7. Kawai A, Muschler GF, Lane JM, et al. Prosthetic knee replacement after resection of a malignant tumor of the distal part of the femur. Medium to long-term results. J Bone Joint Surg Am. 1998;80(5):636-47.

8. Natarajan MV, Sivaseelam A, Ayyappan S, et al. Distal femoral tumours treated by resection and custom mega-prosthetic replacement. Int Orthop. 2005;29(5):309-13. https://doi.org/10.1007/s00264-005-0677-z.

9. Sevelda F, Waldstein W, Panotopoulos J, et al. Survival, failure modes and function of combined distal femur and proximal tibia reconstruction following tumor resection. Eur J Surg Oncol. 2017;43(2):416-22. https://doi. org/10.1016/j.ejso.2016.11.005.

10. Dean B, Whitwell D. (i) Epidemiology of bone and soft-tissue sarcomas. Orthop Trauma. 2009;23:223-30. https://doi.org/10.1016/j.mporth.2009.05.006.

11. Ferreira N, Marais LC, Pillay Y. Primary malignant bone tumours: Epidemiological data from an orthopaedic oncology unit in South Africa. S Afr Orthop J. 2016;15(4):12-16. https://doi.org/10.17159/2309-8309/2016/v15n4a1.

12. Arora RS, Alston RD, Eden TO, et al. The contrasting age-incidence patterns of bone tumours in teenagers and young adults: Implications for aetiology. Int $\mathrm{J}$ Cancer. 2012;131(7):1678-85. https://doi.org/10.1002/ijc.27402.

13. Rodriguez-Merchan EC, Liddle AD. Joint preservation in the adult knee. Cham, Switzerland: Springer; 2017. https://doi.org/10.1007/978-3-319-41808-7.

14. Nie Z, Peng H. Osteosarcoma in patients below 25 years of age: An observational study of incidence, metastasis, treatment and outcomes. Oncol Lett. 2018;16(5):6502-14. https://doi.org/10.3892/ol.2018.9453.

15. Sangle NA, Layfield LJ. Telangiectatic osteosarcoma. Arch Pathol Lab Med. 2012;136(5):572-76. https://doi.org/10.5858/arpa.2011-0204-RS.

16. Huvos AG, Rosen G, Bretsky SS, Butler A. Telangiectatic osteogenic sarcoma: A clinicopathologic study of 124 patients. Cancer. 1982;49(8):1679-89. https:// doi.org/10.1002/1097-0142(19820415)49:8<1679::aid-cncr2820490824>3.0.c 0;2-2.

17. Foundation for Orthopaedic Research Education [Internet]. Outcomes of the quadriceps-sparing lateral approach to the distal femur for tumor resection and endoprosthetic reconstruction. 2016. Available from: https://foreonline.org/ ord-2016/. Accessed 18 Jun 2019. 